\title{
Unsteady isothermal flow behind a magnetogasdynamic shock wave in a self-gravitating gas with exponentially varying density
}

\author{
G. Nath
}

Received: 17 January 2014/ Accepted: 8 May 2014/Published online: 5 June 2014

(C) The Author(s) 2014. This article is published with open access at Springerlink.com

\begin{abstract}
The propagation of spherical (or cylindrical) shock wave in an ideal gas with or without gravitational effects in the presence of a constant azimuthal magnetic field is investigated. Non-similarity solutions are obtained for isothermal flow between the shock and the piston. The numerical solutions are obtained using the Runge-Kutta method of the fourth order. The density of the gas is assumed to be varying and obeying an exponential law. The shock wave moves with variable velocity, and the total energy of the wave is non-constant and varies with time. The effects of variation of the Alfven-Mach number, gravitational parameter and time are obtained. It is investigated that the presence of gravitational field reduces the effect of the magnetic field. Also, the presence of gravitational field increases the compressibility of the medium, due to which it is compressed and, therefore, the distance between the inner contact surface and the shock surface is reduced. The shock waves in conducting perfect gas can be important for description of shocks in supernova explosions, in the study of central part of star burst galaxies, nuclear explosion, rupture of a pressurized vessel and explosion in the ionosphere. Other potential applications of this study include analysis of data from exploding wire experiments and cylindrically symmetric hypersonic flow problems associated with meteors or re-entry vehicles etc. A comparison is made between the solutions in the cases of the gravitating and the non-gravitating medium with or without magnetic field. The obtained solutions are applicable for arbitrary values of time.
\end{abstract}

G. Nath ( $\square)$

Department of Mathematics, Motilal Nehru National Institute of Technology, Allahabad 211004, India

e-mail: gn_chaurasia_univgkp@yahoo.in; gnath@mnnit.ac.in
Keywords Shock wave $\cdot$ Magnetogasdynamics and Electrofluid mechanics · Gravitational effects · Numerical solution · Non-similarity solution

\section{Introduction}

Shock phenomena such as a global shock resulting from a stellar pulsation or supernova explosion passing outward through a stellar envelope or perhaps a shock emanating from a point source such as a man-made explosion in the Earth's atmosphere or an impulsive flare in the Sun's atmosphere have tremendous importance in astrophysics and space sciences. Shock waves are common in the interstellar medium because of a great variety of supersonic motions and energetic events, such as cloud-cloud collision, bipolar outflow from young protostellar objects, powerful mass losses by massive stars in a late stage of their evolution (stellar winds), supernova explosions, central part of star burst galaxies, etc. Shock waves are also associated with spiral density waves, radio galaxies and quasars. Similar phenomena also occur in laboratory situations, for example, when a piston is driven rapidly into a tube of gas (a shock tube), when a projectile or aircraft moves supersonically through the atmosphere, in the blast wave produced by a strong explosion, or when rapidly flowing gas encounters a constriction in a flow channel or runs into a wall.

The analysis and explanation for the internal motion in stars is one of the basic problems in astrophysics. According to the observational data, the unsteady motion of a large mass of gas followed by sudden release of energy results flare-ups in novae and supernovae. A qualitative behavior of the gaseous mass may be discussed with the help of the equations of motion and equilibrium taking 
gravitational forces into account. Numerical solutions for self-similar adiabatic flows in self-gravitating gas were obtained by Sedov [1] and Carrus et al. [2], independently. Purohit [3] and Singh and Vishwakarma [4] have discussed homothermal flows behind a spherical shock wave in a self-gravitating gas using similarity method. Nath et al. [5] have studied the above problem assuming the flow to be adiabatic and self-similar and obtained the effects of the presence of a magnetic field. Shock waves through a variable-density medium have been treated by Sedov [1], Sakurai [6], Nath [7], Rogers [8], Rosenau and Frankenthal [9], Nath et al. [5], Vishwakarma and Yadav [10] and others. Their results are more applicable to the shock formed in the deep interior of stars.

Laumbach and Probstein [11], Hayes [12], Deb Ray [13], Verma and Vishwakarma [14, 15], Vishwakarma [16], Nath [17] have discussed the propagation of shock waves in a medium where density varies exponentially and obtained similarity and non-similarity solutions. These authors have not taken into account the effects of the selfgravitation of the medium. Also, Nath et al. [18] analyzed the non-similarity solutions and investigated the effects of the presence of an ambient azimuthal magnetic field and the self-gravitation of the ambient medium on the flow field behind a magnetogasdynamic spherical (or cylindrical) shock wave in the case of adiabatic flow. They assumed that the density in the ambient medium varies according to exponential law and the initial magnetic field to be constant.

Since at high temperatures that prevail in the problems associated with the shock waves a gas is ionized, electromagnetic effects may also be significant. A complete analysis of such a problem should, therefore, consist of the study of the gasdynamic flow and the electromagnetic field simultaneously. A detailed study towards gaining a better understanding of the interaction between gasdynamic motion of an electrically conducting medium and magnetic field within the context of hyperbolic system has been carried out by many investigators such as Korobeinikov [19], Shang [20], Lock and Mestel [21]. A detailed review in the field of magnetogasdynamic flows can be seen in the paper Shang [20]. Lock and Mestel [21] analyzed the annular self-similar solutions in ideal magnetogasdynamics by casting the ideal magnetogasdynamic equations to a three-dimensional autonomous system in which either the magnetic pressure or the fluid pressure vanishes.

The shock waves in the presence of a magnetic field in conducting perfect gas can be important for description of shocks in supernova explosions and explosion in the ionosphere. The strong magnetic fields play significant roles in the dynamics of the interstellar medium. Among the industrial applications involving applied external magnetic fields are drag reduction in duct flows, design of efficient coolant blankets in tokamak fusion reactors, control of turbulence of immersed jets in the steel casting process and advanced propulsion and flow control schemes for hypersonic vehicles. The magnetic fields have important roles in a variety of astrophysical situations. Complex filamentary structures in molecular clouds, shapes and the shaping of planetary nebulae, synchrotron radiation from supernova remnants, magnetized stellar winds, galactic winds, gamma-ray bursts, dynamo effects in stars, galaxies, and galaxy clusters as well as other interesting problems all involve magnetic fields (see Hartmann [22], Balick and Frank [23]).

In all of the works mentioned above, the effect of selfgravitation and magnetic field is not taken into account by any of the authors in the case of isothermal flow with exponentially varying density.

The purpose of this study is, therefore, to obtain the nonsimilarity solutions for the unsteady isothermal flow behind magnetogasdynamic spherical (or cylindrical) shock waves in a self-gravitating gas with exponentially varying density. The density in the medium ahead of the shock is assumed to obey an exponential law. The medium is assumed to be a perfect gas and the initial magnetic field to be constant. The assumption of isothermal flow is physically realistic, when radiation heat transfer effects are implicitly present. As the shock propagates, the temperature behind it increases and becomes very large so that there is intense transfer of energy by radiation. This causes the temperature gradient to approach zero, that is the dependent temperature tends to become uniform behind the shock front and the flow becomes isothermal (Laumbach and Probstein [24], Sachdev and Ashraf [25], Korobeinikov [19], Zhuravskaya and Levin [26], Nath [7, 17, 27]). A detailed mathematical theory of one-dimensional isothermal blast waves in a magnetic field was developed by Lerche [28, 29]. Numerical solutions for the flow field between the shock and the piston are obtained in the case of isothermal flow in "Solution to the equations". Effects of viscosity and rotation are not taken into account. The present study can be important to verify the accuracy of the solution obtained by the theory of self-similarity and computational methods such as finite difference scheme, finite element, etc.

Variation of the flow variables behind the shock for different values of the Alfven-Mach number, gravitational parameter and time is obtained. It is investigated that the presence of gravitational field reduces the effects of the magnetic field. Also, the presence of gravitational field increases the compressibility of the medium, due to which it is compressed and, therefore, the distance between the inner contact surface and the shock surface is reduced. Further, it is investigated that due to an increase in the strength of magnetic field, the distance of the inner contact surface from the shock front is increased, i.e., the flow field 
behind the shock becomes somewhat rarefied, i.e., there is a decrease in the shock strength. A comparison is made between the solutions in the cases of the self-gravitating and the non-gravitating medium for both the magnetic and non-magnetic cases. The obtained solutions are applicable for arbitrary values of time.

\section{Equations of motion and boundary conditions}

The fundamental equations governing the unsteady and cylindrically (or spherically) symmetric isothermal flow of an electrically conducting and self-gravitating ideal gas, in the presence of an azimuthal magnetic field, may be written as (c.f. Carrus et al. [2], Laumbach and Probstein [24], Sachdev and Ashraf [25], Korobeinikov [19], Zhuravskaya and Levin [26], Nath [7, 27], Whitham [30])

$\frac{\partial \rho}{\partial t}+u \frac{\partial \rho}{\partial r}+\rho \frac{\partial u}{\partial r}+\frac{i u \rho}{r}=0$,

$\frac{\partial u}{\partial t}+u \frac{\partial u}{\partial r}+\frac{1}{\rho}\left[\frac{\partial p}{\partial r}+\mu h \frac{\partial h}{\partial r}+\frac{\mu h^{2}}{r}\right]+\frac{G m}{r^{i}}=0$,

$\frac{\partial h}{\partial t}+u \frac{\partial h}{\partial r}+h \frac{\partial u}{\partial r}+(i-1) \frac{h u}{r}=0$,

$\frac{\partial m}{\partial r}=2 \pi i \rho r^{i}$

$\frac{\partial T}{\partial r}=0$,

where $r$ and $t$ are independent space and time coordinates, $u$ is the fluid velocity, $\rho$ is the density, $p$ is the pressure, $h$ is the azimuthal magnetic field, $T$ is the temperature, $\mu$ is the magnetic permeability, $m$ is the mass contained in a unit cylinder of radius $r$ or in a sphere of radius $r$ and the dimension of $m$ is taken as $[m]=M L^{(i-2)}$ and $i$ takes the values 2 and 1 for the respective cases of spherical and cylindrical symmetries, and $G$ is the gravitational constant. In the non-gravitating case, Eq. (2.4) and the term $\frac{G m}{r^{i}}$ in Eq. (2.2) do not occur. The electrical conductivity of the gas is assumed to be infinite. Therefore, the diffusion term from the magnetic field equation is omitted, and the electrical resistivity is ignored. Also, the effect of viscosity on the flow of the gas is assumed to be negligible.

The above system of equations should be supplemented with an equation of state. An ideal gas behavior of the medium is assumed, so that

$p=\Gamma \rho T ; \quad e=\frac{p}{(\gamma-1) \rho}$,

where $\Gamma$ is the gas constant and $\gamma$ is the ratio of specific heats at constant pressure and volume.

The ambient density of the medium is assumed to obey the exponential law, namely, $\rho_{1}=\rho_{0} e^{\delta R}$,

where ' $R$ ' is the shock radius and $\rho_{0}$ and $\delta$ are suitable constants and the subscript ' 1 ' refers to the conditions immediately ahead of the shock.

We assume that a strong spherical (or cylindrical) shock is propagating outwardly in the undisturbed ideal gas with infinite electrical conductivity and variable density in the presence of a constant azimuthal magnetic field. The jump conditions at the shock wave are given by the principles of conservation of mass, momentum, magnetic field and energy across the shock (see [7, 17, 27, 30]), namely

$$
\begin{aligned}
& \rho_{1} V=\rho_{2}\left(V-u_{2}\right), \\
& h_{1} V=h_{2}\left(V-u_{2}\right), \\
& p_{1}+\frac{1}{2} \mu h_{1}^{2}+\rho_{1} V^{2}=p_{2}+\frac{1}{2} \mu h_{2}^{2}+\rho_{2}\left(V-u_{2}\right)^{2}, \\
& e_{1}+\frac{p_{1}}{\rho_{1}}+\frac{1}{2} V^{2}+\frac{\mu h_{1}^{2}}{\rho_{1}}-\frac{F_{1}}{\rho_{1} V} \\
& =e_{2}+\frac{p_{2}}{\rho_{2}}+\frac{1}{2}\left(V-u_{2}\right)^{2}+\frac{\mu h_{2}^{2}}{\rho_{2}}-\frac{F_{2}}{\rho_{1} V}, \\
& m_{1}=m_{2},
\end{aligned}
$$

where the subscript ' 2 ' denotes the conditions immediately behind the shock front, $V\left(=\frac{d R}{d t}\right)$ denotes the velocity of the shock front and ' $F$ ' is the radiation heat flux.

If the shock is a strong one, then the jump conditions (2.8) become

$$
\begin{aligned}
& u_{2}=(1-\beta) V, \\
& \rho_{2}=\frac{\rho_{1}}{\beta}, \\
& p_{2}=L \rho_{1} V^{2}, \\
& m_{2}=m_{1}, \\
& h_{2}=\frac{h_{1}}{\beta},
\end{aligned}
$$

where $L=\left[(1-\beta)+\frac{1}{2 M_{A}^{2}}\left(1-\frac{1}{\beta^{2}}\right)\right]$, and $M_{A}=\left(\frac{\rho_{1} V^{2}}{\mu h_{1}^{2}}\right)^{\frac{1}{2}}$ is the Alfven-Mach number. The density ratio $\beta(0<\beta<1)$ across the shock front is obtained by the relation

$$
\begin{aligned}
\beta^{2}- & \beta\left(\frac{\gamma\left(1+M_{A}^{-2}\right)-1}{(\gamma+1)}\right)+\frac{(\gamma-2) M_{A}^{-2}}{(\gamma+1)} \\
- & \frac{2(\gamma-1) \beta\left(F_{2}-F_{1}\right)}{p_{2} V(1-\beta)(\gamma+1)}=0 .
\end{aligned}
$$

As the shock is strong one, we assume $\left(F_{2}-F_{1}\right)$ to be negligible in comparison with $\rho_{1} V^{3}$, i.e., with the product of $p_{2}$ and $V$ (Laumbach and Probstein [24], Nath [7, 17, 27]). Therefore, (2.10) reduces to 
$\beta^{2}-\beta\left(\frac{\gamma\left(1+M_{A}^{-2}\right)-1}{(\gamma+1)}\right)+\frac{(\gamma-2) M_{A}^{-2}}{(\gamma+1)}=0$.

Equation (2.5) together with Eq. (2.6) gives

$\frac{p}{p_{2}}=\frac{\rho}{\rho_{2}}$.

Let the solution of Eqs. (2.1)-(2.6) be of the form (Verma and Vishwakarma [14], Vishwakarma [16], Nath [17], Nath et al. [18])

$u=\frac{1}{t} U(\eta)$,

$\rho=t^{\Omega} D(\eta)$,

$p=t^{\Omega-2} P(\eta)$,

$\sqrt{\mu} h=t^{(\Omega-2) / 2} H(\eta)$,

$m=t^{\Omega} K(\eta)$,

where

$\eta=t e^{\lambda r}, \lambda \neq 0$

and the constants $\Omega$ and $\lambda$ are to be determined subsequently. We choose the shock surface to be given by

$\eta_{0}=$ constant.

The variable $\eta$ assumes constant value $\eta_{0}$ at the shock surface, so that its velocity is given by

$V=-\frac{1}{\lambda t}$

which represents an outgoing shock surface, if $\lambda<0$.

The solution of Eqs. (2.1)-(2.5) in the form (2.13) to (2.16) is compatible with the shock conditions, if

$\Omega=2, \quad \lambda=-\frac{\delta}{2}$.

Since necessarily $\lambda<0$, relation (2.17) shows that $\delta>0$, thereby meaning that the shock surface expands outwardly in an exponentially increasing medium (Hayes [12], Deb Ray [13]).

From Eqs. (2.16) and (2.17), we obtain
$R=\frac{2}{\delta} \log \left(\frac{t}{t_{0}}\right)$

where $t_{0}$ is the duration of the almost instantaneous explosion.

\section{Solution to the equations}

The flow variables in the flow field behind the shock front will be obtained by solving the Eqs. (2.1)-(2.5). From Eqs. (2.13), (2.16) and (2.17), we obtain

$\frac{\partial u}{\partial t}=\lambda u V-V \frac{\partial u}{\partial r}$

$\frac{\partial \rho}{\partial t}=-2 \rho \lambda V-V \frac{\partial \rho}{\partial r}$,

$\frac{\partial p}{\partial t}=-V \frac{\partial p}{\partial r}$

$\frac{\partial h}{\partial t}=-V \frac{\partial h}{\partial r}$

$\frac{\partial m}{\partial t}=-2 \lambda m V-V \frac{\partial m}{\partial r}$.

Using the Eqs. (3.1)-(3.5) and the transformations $r^{\prime}=\frac{r}{R}, \quad u^{\prime}=\frac{u}{V}, \quad \rho^{\prime}=\frac{\rho}{\rho_{2}}, \quad h^{\prime}=\frac{h}{h_{2}}, \quad p^{\prime}=\frac{p}{p_{2}}$,

$$
m^{\prime}=\frac{m}{m_{2}}
$$

in the fundamental Eqs. (2.1)-(2.4) and (2.12), we obtain for spherical symmetry $(i=2)$

$p^{\prime}=\rho^{\prime}$,

$\frac{d \rho^{\prime}}{d r^{\prime}}=\frac{\rho^{\prime}}{\left(1-u^{\prime}\right)}\left[\frac{d u^{\prime}}{d r^{\prime}}+2 \log \left(\frac{t}{t_{0}}\right)+\frac{2 u^{\prime}}{r^{\prime}}\right]$,

$\frac{d h^{\prime}}{d r^{\prime}}=\frac{h^{\prime}}{\left(1-u^{\prime}\right)}\left[\frac{d u^{\prime}}{d r^{\prime}}+\frac{u^{\prime}}{r^{\prime}}\right]$

$\frac{d m^{\prime}}{d r^{\prime}}=\frac{4 \rho^{\prime} r^{\prime 2}\left(\log \frac{t}{t_{0}}\right)^{3}}{\beta\left[2\left(\log \frac{t}{t_{0}}\right)^{2}-2\left(\log \frac{t}{t_{0}}\right)+1\right]}$ 
where $L^{*}=\pi \rho_{0} G t_{0}^{2}$ is the gravitational parameter.

Also, the total energy of the disturbance is given by

$E=2 \pi i \int_{\bar{r}}^{R} \rho\left[e+\frac{1}{2} u^{2}+\frac{\mu h^{2}}{2 \rho}-\frac{G m}{r^{i-1}}\right] r^{i} d r$,

where $\bar{r}$ is the position of inner boundary of the disturbance. Using (2.6), (2.9) and (3.6), (3.12) becomes (for $i=2$ )

$E=\frac{16 \pi \rho_{0}}{\delta^{2} \eta_{0}^{2}} R^{3} J$

where

$J=\int_{\frac{r^{\prime}}{r^{\prime}}}^{1}\left[\frac{L p^{\prime}}{(\gamma-1)}+\frac{\rho^{\prime} u^{\prime 2}}{2 \beta}+\frac{M_{A}^{-2} h^{\prime 2}}{2 \beta}-\pi G \eta_{0}^{2} \frac{m^{\prime}}{r^{\prime}\left(\log \frac{t}{t_{0}}\right)}\left\{2\left(\log \frac{t}{t_{0}}\right)^{2}\right.\right.$ $\left.\left.-2\left(\log \frac{t}{t_{0}}\right)+1\right\}\left(\frac{t}{t_{0}}\right)^{2}\right] r^{\prime 2} d r^{\prime}$

Thus, the total energy of the shock wave is non-constant and varies with time. The increase of total energy may be achieved by the pressure exerted on the fluid by the inner expanding surface (a contact surface or a piston). A situation very much of the same kind may prevail during the formation of a cylindrical spark channel from exploding wires. In addition, in the usual cases of spark breakdown, time-dependent energy input is a more realistic assumption than instantaneous energy input (see [31, 32]).

In terms of dimensionless variables $r^{\prime}, \rho^{\prime}, p^{\prime}, h^{\prime}, u^{\prime}$ and $m^{\prime}$, the shock conditions (2.9) take the form

$r^{\prime}=1, \quad \rho^{\prime}=1, \quad p^{\prime}=1, \quad h^{\prime}=1, \quad u^{\prime}=(1-\beta)$, $m^{\prime}=1$.

Equations (3.7)-(3.11) along with the boundary conditions (3.14) give the solution of our problem. The solution so obtained is a non-similar one, since the motion behind the shock can be determined only when a definite value for time is prescribed.

\section{Results and discussion}

The distribution of the flow variables behind the shock front in the case of isothermal flow is obtained from Eqs. (3.7) to (3.11) with the boundary conditions (3.14) by the RungeKutta method of the fourth order. For the purpose of numerical integration, the values of the constant parameters are taken to be (Pai [33], Nath [7, 27], Rosenau [34], Nath et al. [18]) $\gamma=\frac{5}{3} ; M_{A}^{-2}=0,0.04,0.08,0.1 ; t / t_{0}=2,3$; $L^{*}=0,0.1$. For fully ionized gas, $\gamma=\frac{5}{3}$, and therefore, it is applicable to the stellar medium. Rosenau and Frankenthal
[8] have shown that the effects of magnetic field on the flow field behind the shock are significant when $M_{A}^{-2} \geq 0.01$; therefore, the above values of $M_{A}^{-2}$ are taken for calculation in the present problem. The value $M_{A}^{-2}=0$ corresponds to the non-magnetic case. The value $L^{*}=0, M_{A}^{-2}=0$ corresponds to the solution in the non-gravitating and non-magnetic case (the solution obtained by Nath [17] in the dust-free isothermal case). Our present study is the generalization of earlier works of Nath [17] in the case of non-dusty isothermal flow by considering the presence of gravitational force and the presence of azimuthal magnetic field. It should be noted that $0<\frac{t}{t_{0}}<1$ corresponds to the shock free flow and $1<\frac{t}{t_{0}}<\infty$ corresponds to the flow under the influence of shock (i.e., shock formation requires that $\frac{t}{t_{0}}>1$ ); therefore, the above values of $\frac{t}{t_{0}}$ are taken for calculation to know the flow field behind the shock at different times. Starting from the shock front the numerical integration is carried out until the singularity of the solution

$\rho^{\prime}\left\{(1-\beta)+\frac{M_{A}^{-2}}{2}\left(1-\frac{1}{\beta^{2}}\right)\right\}+\frac{M_{A}^{-2} h^{\prime}}{\beta}-\rho^{\prime}\left(1-u^{\prime}\right)^{2}=0$

is reached. This marks the inner boundary of the disturbance and at this surface the value of $r^{\prime}\left(=\overline{r^{\prime}}\right)$ remains constant. The inner boundary is the position in the flow field behind the shock front at which the velocity of the inner boundary and the fluid velocity are equal.

The results are shown in Figs. $1 \mathrm{a}-\mathrm{c}, 2$ and Table 1. These figures and table show that the self-gravitation of the medium has a significant effect on the flow variables and shock strength. Our analysis reveals that after inclusion of gravitational field effect, surprisingly the shock strength increases and the effect of magnetic field on the shock decreases and remarkable difference is found in the distribution of the flow variables.

Table 1 shows the variation of the density ratio $\beta\left(=\frac{\rho_{1}}{\rho_{2}}\right)$ across the shock front and the position of the inner expanding surface for different values of $M_{A}^{-2}$ with $\gamma=\frac{5}{3}$; $t / t_{0}=2,3$; and $L^{*}=0,0.1$ in both the gravitating and non-gravitating cases. The shock strength decreases with an increase in the strength of the magnetic field. By an increase in time the distance of the inner contact surface from the shock front is decreased for gravitating and magnetic or non-magnetic case (i.e., for $L^{*}=0.1$, $M_{A}^{-2}=0$ or 0.1$)$ and for non-gravitating and non-magnetic case (i.e., for $L^{*}=0, M_{A}^{-2}=0$ ). It means that the shock becomes stronger with time in gravitating and magnetic or non-magnetic case, and in non-gravitating and non-magnetic case, but reverse behavior is obtained for non-gravitating and magnetic case (i.e., for $L^{*}=0, M_{A}^{-2} \neq 0$ ), i.e., the shock strength decreases with time in non-gravitating 
Fig. 1 Variation of the flow variables in the region behind the shock front for $\gamma=\frac{5}{3} .1$ $M_{A}^{-2}=0, \frac{t}{t_{0}}=2 ; 2 M_{A}^{-2}=0.04$, $\frac{t}{t_{0}}=2 ; 3 M_{A}^{-2}=0.1, \frac{t}{t_{0}}=2 ; 4$ $M_{A}^{-2}=0, \frac{t}{t_{0}}=3 ; 5 M_{A}^{-2}=0.04$, $\frac{t}{t_{0}}=3 ; 6 M_{A}^{-2}=0.1, \frac{t}{t_{0}}=3$. a reduced fluid velocity $u^{\prime}$ b reduced density $\rho^{\prime}$ (or pressure $p^{\prime}$ ). c reduced mass $m^{\prime}$ a

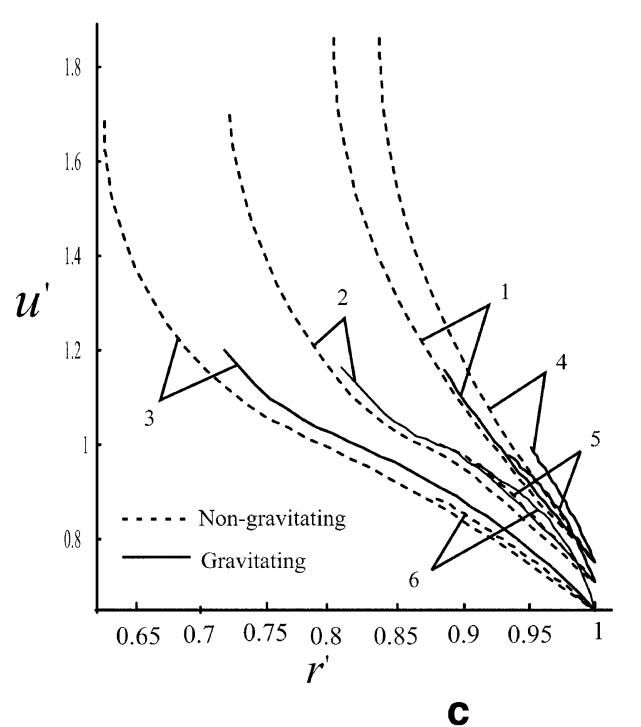

b

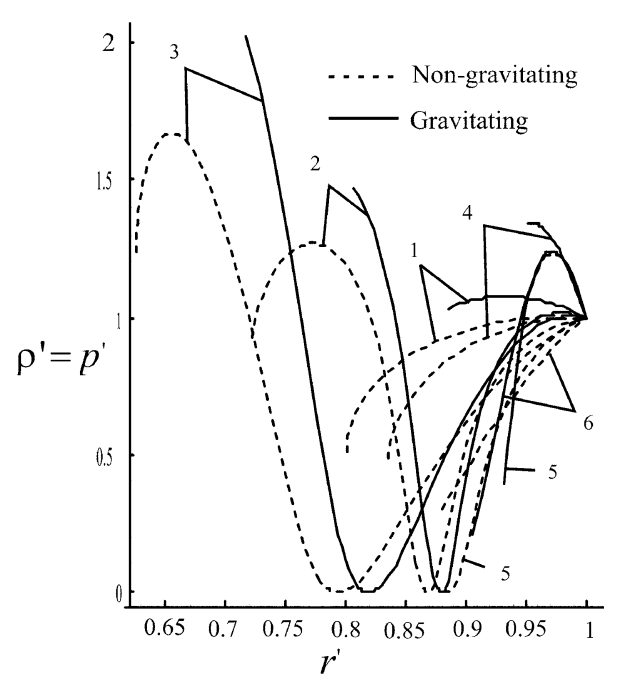

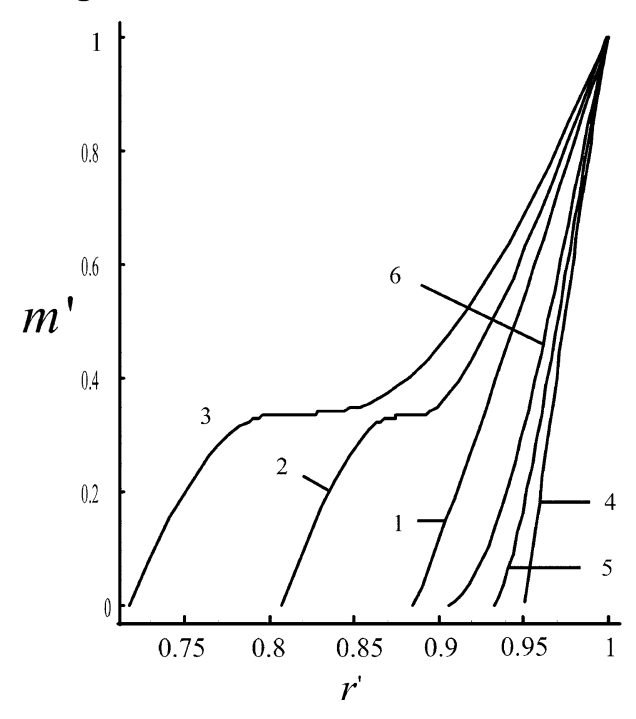

and non-magnetic case. Also, Table 1 shows that the distance of the inner expanding surface from the shock front is less in the case of the gravitating medium in comparison with that in the case of the non-gravitating medium. Physically, it means that the gas behind the shock is compressed in the gravitating medium, that is, the shock strength is increased in the gravitating medium.

Figures $1 \mathrm{a}-\mathrm{c}$ and 2 show the variation of the flow variables $u^{\prime}, \rho^{\prime}\left(=p^{\prime}\right), m^{\prime}$ and $h^{\prime}$ with $r^{\prime}$ at various values of the parameters $M_{A}^{-2}, t / t_{0}$ and $L^{*}$.

Figure 1a, c shows the distributions of reduced velocity and reduced mass, respectively. These figures show that the reduced velocity increases, while reduced mass increases as we move from the shock front to the inner expanding surface (or piston). The velocity increases from the shock front and approaches to maximum near the inner expanding surface

(see Fig. 1a). In fact the velocity of the inner expanding surface is higher than the fluid velocity just behind the shock due to increasing energy input given by Eq. (3.13).

Figure 2 shows that the reduced magnetic field $h^{\prime}$ increases as we move from the shock front to the inner expanding surface and its profiles decrease after attaining a maximum for $t / t_{0}=2$ in the flow field region behind the shock front. This behavior of magnetic field is obtained due to less compression of magnetic flux which leads to decrease in magnetic field $h^{\prime}$.

\section{Conclusions}

Non-similarity solution for propagation of explosion waves in a stellar model in the case of unsteady isothermal flow, 


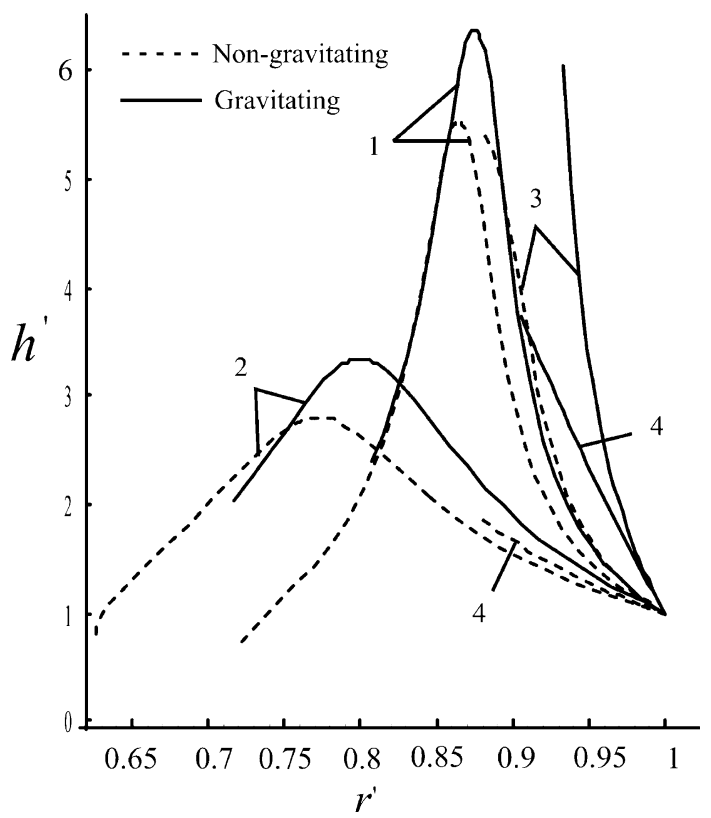

Fig. 2 Variation of reduced azimuthal magnetic field $h^{\prime}$ in the region behind the shock front for $\gamma=\frac{5}{3} .1 M_{A}^{-2}=0.04, \frac{t}{t_{0}}=2 ; 2 M_{A}^{-2}=0.1$, $\frac{t}{t_{0}}=2 ; 3 M_{A}^{-2}=0.04, \frac{t}{t_{0}}=3 ; 4 M_{A}^{-2}=0.1, \frac{t}{t_{0}}=3$

in which density varies exponentially, has been obtained in this paper. The shock wave moves with variable velocity, and the total energy of the wave is not constant and varies with time. It is investigated that the presence of gravitational field reduces the effects of the magnetic field. Also, the presence of gravitational field increases the compressibility of the medium, due to which it is compressed and, therefore, the distance between the inner contact surface and the shock surface is reduced.

The article concerns with the explosion problem; however, the methodology analysis presented here may be used to describe many other physical systems involving non-linear hyperbolic partial differential equations. The shock waves in conducting perfect gas can be important for description of shocks in supernova explosions, in the study of central part of star burst galaxies, nuclear explosion, rupture of a pressurized vessel and explosion in the ionosphere. Other potential applications of this study include analysis of data from exploding wire experiments and cylindrically symmetric hypersonic flow problems associated with meteors or reentry vehicles (c.f. Hutchens [35]). Also, the present study can be important to verify the accuracy of the solution obtained by the theory of self-similarity and computational methods such as finite difference scheme, finite element, etc.

The following conclusions may be drawn from the finding of the current analysis (see Figs. 1a-c, 2; Table 1):

1. By an increase in the strength of magnetic field, the distance of the inner contact surface from the shock front is increased in general, i.e., the flow field behind the shock becomes somewhat rarefied, i.e., there is a decrease in the shock strength.

2. Due to the presence of gravitation, the pressure (density), velocity and azimuthal magnetic field increase, in general, as we move inward from the shock front.

3. It is found that the reduced velocity and reduced pressure (density) decrease in general with the strength of magnetic field, whereas the reduced mass shows a reverse behavior.

4. The reduced pressure (density) with or without magnetic field in non-gravitating case (i.e., for $L^{*}=0$ ) decreased with an increase in time $\left(t / t_{0}\right)$ in general, whereas it increases in gravitating case (i.e., for $\left.L^{*}=0.1\right)$.

5. It is found that reduced velocity and reduced magnetic field increase with an increase in time $\left(t / t_{0}\right)$, whereas the reduced mass shows a reverse behavior.

Table 1 Variation of the density ratio $\beta\left(=\frac{\rho_{1}}{\rho_{2}}\right)$ across the shock front and the position of the inner expanding surface $\overline{r^{\prime}}$ for different values of $M_{A}^{-2}$ with $\gamma=\frac{5}{3}$

\begin{tabular}{|c|c|c|c|c|c|}
\hline \multirow[t]{3}{*}{$M_{A}^{-2}$} & \multirow[t]{3}{*}{$\beta$} & \multicolumn{4}{|c|}{ Position of the expanding surface $\overline{r^{\prime}}$} \\
\hline & & \multicolumn{2}{|l|}{$t / t_{0}=2$} & \multicolumn{2}{|l|}{$t / t_{0}=3$} \\
\hline & & Gravitating case $\left(L^{*}=0.1\right)$ & Non-gravitating case $\left(L^{*}=0\right)$ & Gravitating case $\left(L^{*}=0.1\right)$ & Non-gravitating case $\left(L^{*}=0\right)$ \\
\hline 0 & 0.25 & 0.951519 & 0.835882 & 0.957585 & 0.866180 \\
\hline 0.01 & 0.261039 & 0.9464 & 0.9237 & 0.9840 & 0.846608 \\
\hline 0.04 & 0.292116 & 0.93256 & 0.8820 & 0.9826 & 0.783417 \\
\hline 0.08 & 0.330278 & 0.9194 & 0.8255 & 0.9810 & 0.65622 \\
\hline 0.1 & 0.34838 & 0.9060 & 0.800079 & 0.9798 & 0.646472 \\
\hline
\end{tabular}


Acknowledgments The author is grateful to Dr. J. P. Vishwakarma, Professor of Mathematics DDU Gorakhpur University Gorakhpur273009, India, for his many useful suggestions and discussions.

Open Access This article is distributed under the terms of the Creative Commons Attribution License which permits any use, distribution, and reproduction in any medium, provided the original author(s) and the source are credited.

\section{References}

1. Sedov, L.I.: Similarity and dimensional methods in mechanics. Academic Press, New York (1959)

2. Carrus, P.A., Fox, P.A., Hass, F., Kopal, Z.: Propagation of shock waves in the generalized Roche model. Astrophys. J. 113, 193-209 (1951)

3. Purohit, S.C.: Self-similar homothermal flow of self-gravitating gas behind shock wave. J. Phys. Soc. (Jpn) 36, 288-292 (1974)

4. Singh, J.B., Vishwakarma, P.R.: Self-similar solutions in the theory of flare-ups in novae I. Astrophys. Space Sci. 95, 99-104 (1983)

5. Nath, O., Ojha, S.N., Takhar, H.S.: A study of stellar point explosion in a self-gravitating radiative magnetohydrodynamic medium. Astroph. Space Sci. 95, 99-104 (1983)

6. Sakurai, A.: Propagation of spherical shock waves in stars. J. Fluid Mech. 1, 436-453 (1956)

7. Nath, G.: Magnetogasdynamic shock wave generated by a moving piston in a rotational axisymmetric isothermal flow of perfect gas with variable density. Adv. Space Res. 47, 1463-1471 (2011)

8. Rogers, M.H.: Analytic solutions for blast wave problem with an atmosphere of varying density. Astrophys. J. 125, 478-493 (1957)

9. Rosenau, P., Frankenthal, S.: Equatorial propagation of axisymmetric magnetohydrodynamic shocks I. Phys. Fluids 19, 1889-1899 (1976)

10. Vishwakarma, J.P., Yadav, A.K.: Self-similar analytical solutions for blast waves in inhomogeneous atmospheres with frozen-inmagnetic field. Eur. Phys. J. B 34, 247-253 (2003)

11. Laumbach, D.D., Probstein, R.F.: A point explosion in a cold exponential atmosphere part I. J. Fluid Mech. 35, 53-75 (1968)

12. Hayes, W.D.: Self-similar strong shocks in an exponential medium. J. Fluid Mech. 32, 305-315 (1968)

13. Deb Ray, G.: An exact analytic solution for strong plane shock waves in an exponential medium. Bull. Cal. Math. Soc. 66, 27-31 (1974)

14. Verma, B.G., Vishwakarma, J.P.: Propagation of magnetogasdynamic plane shock waves in an exponential medium. Nuovo Cimento 32, 267-272 (1976)

15. Verma, B.G., Vishwakarma, J.P.: Axially symmetric explosion in magnetogasdynamic. Astrophys. Space Sci. 69, 177-188 (1980)
16. Vishwakarma, J.P.: Propagation of shock waves in a dusty gas with exponentially varying density. Eur. Phys. J. B 16, 369-372 (2000)

17. Nath, G.: Propagation of a strong cylindrical shock wave in a rotational axisymmetric dusty gas with exponentially varying density. Res. Astron. Astrophys. 10, 445-460 (2010)

18. Nath, G., Vishwakarma, J.P., Srivastava, V.K., Sinha, A.K.: Propagation of magnetogasdynamic shock waves in a self-gravitating gas with exponentially varying density. J. Theor. Appl. Phys. 7, 15 (2013)

19. Korobeinikov, V.P.: Problems in the theory of point explosion in gases. In: Proceedings of the Steklov Institute of Mathematics, vol. 119. American Mathematical Society, Providence (1976)

20. Shang, J.S.: Recent research in magneto-aerodynamics. Prog. Aerosp. Sci. 21, 1-20 (2001)

21. Lock, R.M., Mestel, A.J.: Annular self-similar solution in ideal gas magnetogasdynamics. J. Fluid Mech. 74, 531-554 (2008)

22. Hartmann, L.: Accretion Processes in Star Formation. Cambridge University Press, Cambridge (1998)

23. Balick, B., Frank, A.: Shapes and shaping of planetary nebulae. Annu. Rev. Astron. Astrophys. 40, 439-486 (2002)

24. Laumbach, D.D., Probstein, R.F.: Self-similar strong shocks with radiation in a decreasing exponential atmosphere. Phys. Fluids 13, 1178-1183 (1970)

25. Sachdev, P.L., Ashraf, S.: Converging spherical and cylindrical shocks with zero temperature gradient in the rear flow-field. J. Appl. Math. Phys. (ZAMP) 22, 1095-1102 (1971)

26. Zhuravskaya, T.A., Levin, V.A.: The propagation of converging and diverging shock waves under the intense heat exchange conditions. J. Appl. Math. Mech. 60, 745-752 (1996)

27. Nath, G.: Propagation of a cylindrical shock wave in a rotational axisymmetric isothermal flow of a non-ideal gas in magnetogasdynamics. Ain Shams Eng. J. 3, 393-401 (2012)

28. Lerch, I.: Mathematical theory of one-dimensional isothermal blast waves in a magnetic field. Aust. J. Phys. 32, 491-502 (1979)

29. Lerch, I.: Mathematical theory of cylindrical isothermal blast waves in a magnetic field. Aust. J. Phys. 34, 279-301 (1981)

30. Whitham, G.B.: On the propagation of shock waves through regions of non-uniform area or flow. J. Fluid Mech. 4, 337-360 (1958)

31. Freeman, R.A., Craggs, J.D.: Shock waves from spark discharges. J. Phys. D Appl. Phys. 2, 421-427 (1969)

32. Director, M.N., Dabora, E.K.: An experimental investigation of variable energy blast waves. Acta Astronaut. 4, 391-407 (1977)

33. Pai, S.I.: Magnetogasdynamic and Plasma Dynamics. Springer, Wien (1986)

34. Rosenau, P.: Equatorial propagation of axisymmetric magnetohydrodynamic shocks II. Phys. Fluids 20, 1097-1103 (1977)

35. Hutchens, G.J.: Approximate cylindrical blast theory: nearfield solutions. J. Appl. Phys. 77, 2912-2915 (1995) 